\title{
L'incontournable absente. Sur la communication dans les manuels de méthode
}

The Unavoidable Absence: On Communication in Methods Textbooks

Anne Jarrigeon, Emmanuelle Lallement, Jean-Baptiste Perret et Julien Tassel

\section{(2) OpenEdition}

\section{Journals}

Édition électronique

URL : http://journals.openedition.org/edc/149

DOI : $10.4000 /$ edc. 149

ISSN : 2101-0366

Éditeur

Université Lille-3

Édition imprimée

Date de publication : 1 décembre 2004

ISBN : 2-9514961-5-X

ISSN : $1270-6841$

Référence électronique

Anne Jarrigeon, Emmanuelle Lallement, Jean-Baptiste Perret et Julien Tassel, «L'incontournable absente. Sur la communication dans les manuels de méthode », Études de communication [En ligne] 27 | 2004, mis en ligne le 18 novembre 2014, consulté le 21 avril 2019. URL : http:// journals.openedition.org/edc/149; DOI : 10.4000/edc.149

Ce document a été généré automatiquement le 21 avril 2019

(c) Tous droits réservés 


\title{
L'incontournable absente. Sur la communication dans les manuels de méthode
}

The Unavoidable Absence: On Communication in Methods Textbooks

\author{
Anne Jarrigeon, Emmanuelle Lallement, Jean-Baptiste Perret et Julien \\ Tassel
}

1 À observer les rayons des librairies on ne peut qu'être frappé de la place croissante qu'occupent les manuels de méthode depuis quelques années dans l'édition en sciences humaines. Manuels de recherche, guides d'enquête, art de la thèse ou du mémoire se disputent les ventes avec les collections spécialisées en méthodes, de celle du questionnaire à celle de l'observation en passant par l'entretien ou l'analyse de contenu. Si leur nature est variable, tous ces manuels partagent une certaine vocation pédagogique et se présentent comme un outil pour le chercheur néophyte, censé contribuer à la production et à la mise en forme de son travail.

2 Quand on s'intéresse à l'épistémologie des sciences humaines, dans le cadre de l'enseignement de la méthodologie de la recherche ${ }^{1}$, ces manuels représentent une véritable scène d'exposition, de cristallisation et de modélisation des pratiques de recherche. À ce titre, ils constituent un des lieux privilégiés d'observation et d'analyse des représentations de celle-ci. Dans la perspective d'une analyse de la recherche en train de se faire, les manuels permettent en effet de mettre au jour certaines modalités selon lesquelles elle est normalisée, enseignée, transmise et reproduite. Comment la recherche se fabrique t-elle dans les manuels? Quels sont les processus de production du savoir décrits? Comment sont prises en compte les interactions entre le chercheur et «ses objets»? Comment sont présentées les situations de rencontre? Quel est le statut de l'écriture ? Comment est pensé le rapport entre interaction directe et textualisation? Le manuel est-il porteur d'un modèle, voire d'une idéologie, de la pratique de recherche? Autant de questions qui, finalement, désignent la communication comme dimension essentielle de tout processus de recherche. 
3 Par dimension communicationnelle de la recherche, nous entendons que les sciences produisent nécessairement leurs connaissances dans la communication. En effet, on ne perçoit « la réalité » qu'à travers une communication. On ne la «transmet », a fortiori on ne l'étudie ou l'analyse, qu'à travers d'une autre communication. Ces communications ne sont ni neutres, ni transparentes: elles introduisent dans la recherche des problèmes pragmatiques (liés aux places, aux espaces et aux situations d'échanges entre les différents interlocuteurs), sémiotiques (liés aux caractéristiques matérielles des supports et des moyens de ces échanges), poétiques (liés à l'écriture du chercheur, aux modes d'exposition des « résultats »)... Enfin et surtout, la communication est instaurative : elle contribue à construire et transformer la pensée du chercheur. Tous ces éléments ne sont donc pas moins importants que l'ensemble des "biais» (sociaux, de calculs, de représentativité, etc.) que la méthodologie canonique nous a appris à considérer comme des obstacles à une recherche valide en sciences humaines, et ils contribuent bien autant que ces derniers à bâtir « la recherche » et la connaissance qu'elle produit.

Dans cette perspective, l'étude de manuels de recherche en sciences humaines et sociales nous permet de comprendre dans quelle mesure sont pensées, intégrées et manipulées les dimensions communicationnelles inhérentes à toute recherche.

5 Nous nous proposons, à partir de l'analyse d'une vingtaine de manuels rencontrés dans le cadre de notre pratique pédagogique ${ }^{2}$, de saisir des logiques de prise en compte de la communication en les mettant en relation avec les postures épistémologiques implicites contenues dans les manuels. L'hétérogénéité de notre corpus ne nous permet évidemment pas de dresser le portrait unifié de la méthodologie en sciences humaines et sociales. Il ne s'agit pas non plus de critiquer les manuels eux-mêmes, ni de pointer des manques épistémologiques sur un type d'objet dont ce n'est ni la préoccupation ni la vocation première, encore moins de nous ériger en juges d'une méthode communicationnellement correcte. Plus simplement, et à titre de contribution à une réflexion plus large, il nous importe de montrer en quoi le statut accordé à la communication dans les manuels peut apporter des éléments de réflexion sur la nature communicationnelle de la connaissance scientifique en sciences humaines et sociales.

Une première analyse montrera que dans les manuels étudiés, la communication n'est généralement pas complètement évacuée des discours de la méthode. On assiste même à un mouvement croissant de prise en compte de l'épaisseur des interactions entre le chercheur et les autres acteurs sociaux. Pourtant, le discours méthodologique, de par sa vocation même, semble se trouver enfermé dans une position contradictoire : s'ouvrir à la complexité des enjeux communicationnels de la recherche (caractère ouvert des situations d'interaction, relations aux objets, attention portée à la matérialité des documents, rhétorique d'exposition, etc.) tout en réaffirmant les principes d'une épistémologie classique qui assimile volontiers la scientificité des procédures à l'extériorité du chercheur vis-à-vis de ce qu'il étudie. Une analyse de l'objet manuel luimême, en tant qu'objet discursif organisé, permettra dans un second temps de mettre en lumière les tensions épistémologiques à l'œuvre. Ainsi, une observation de la communication de la méthode succédera à l'analyse de la communication dans la méthode. 


\section{La communication entre évocation et conjuration}

7 Tous travaillés par la question de la prise en compte du «off» de la recherche, les manuels de notre corpus dessinent une image contrastée de la communication inhérente à toute méthodologie d'enquête. À prendre au sérieux la diversité des manuels étudiés (manuels se consacrant à une technique de recueil de données comme l'observation ou l'entretien, manuels présentant plutôt une démarche, d'autres une discipline...) il serait assez tentant d'en dresser une typologie: entre ceux qui occultent toute dimension communicationnelle et ceux qui, mettant en avant certains aspects de la communication, font de sa maîtrise une de leurs raisons d'être, il est possible de hiérarchiser les degrés de prise en compte. Ainsi, des manuels plus opérationnels font-ils souvent l'impasse sur ce qui n'est pas directement utilisable dans une méthode présentée comme boîte à outils, axée sur l'efficacité dans la production des résultats et sur la rédaction d'un produit fini : mémoire, rapport, voire thèse. À l'opposé, des manuels plus « réflexifs » font une large part aux problèmes épistémologiques, interrogent ce qu'est la méthode, ce qu'est la discipline concernée, voire ce qu'est la recherche elle-même. Mais malgré ces différences dans le degré de prise en compte, ces manuels semblent avoir en commun des manières de traiter de la dimension communicationnelle des pratiques de recherche. L'épistémologie implicite qui semble commune aux auteurs de manuels manifeste des tendances contradictoires : d'une part, un souci d'homogénéité et d'objectivité qui tend à évacuer la communication comme dimension centrale dans la production du savoir et, d'autre part, l'affirmation pourtant largement partagée que le réel est construit, que la rencontre avec les enquêtés est fondatrice, voire pour certains que le travail d'écriture est constituant et non seulement constitué. Largement évoqué comme inévitable dans le processus de recherche, ce qui relève de la communication donne lieu le plus souvent à des tentatives de maîtrise ou d'évitement. Si bien que la dimension communicationnelle de la recherche, évacuée par la porte, revient par la fenêtre: son traitement obéit à une logique paradoxale d'évocation et de conjuration.

\section{Un enjeu de maîtrise de la communication}

8 La question de la communication apparaît le plus souvent sous la forme d'obstacles à éviter pour garantir une certaine objectivité à la recherche. La méthodologie est souvent présentée comme le moyen de mettre en place des systèmes de protection contre ce qui apparaît comme un bruit à maîtriser dans le déroulement d'une bonne recherche. On y apprend par exemple à vérifier la véracité de la parole des acteurs, à croiser les informations recueillies, à ne pas introduire de biais dans les situations observées. Autant de précautions à prendre censées garantir que la dimension communicationnelle, jamais désignée comme telle, ne nuira pas à la construction du savoir scientifique. Même les manuels sensibles aux questions d'interaction ou s'inscrivant dans une vision constructiviste de la recherche, pensons à certains manuels d'ethnographie comme Le Guide de l'enquête de terrain, insistent sur la nécessité de contrôler tout ce qui ne relèverait pas des opérations délibérées du chercheur.

9 Ce repli de la communication sur l'opératoire et le maîtrisable se manifeste par exemple dans le statut donné au réel. Celui-ci et ses notions satellitaires (information, données, matériau, terrain) sont successivement présentés comme le fruit de l'interaction entre le 
chercheur et son objet de recherche puis considérés comme un prélèvement que le chercheur opère sur la réalité. Que les données soient « recueillies » ou " produites », le réel semble toujours déjà là, il s'agit de le restituer dans sa pureté, sans que cet acte même de recherche ne le modifie. Dans certains cas comme dans Le Guide du mémoire, ce que recouvre le travail de terrain n'est pas explicité et se trouve remplacé par l'idée du stockage d'informations. Ce stockage se fait souvent via la métaphore de la cueillette: dans L'art de l'enquête on cueille et on recueille des données, dans L'enquête ethnologique de terrain, on "récolte des récits de vie " comme si ces derniers avaient une existence en dehors de la situation de communication particulière qu'est la rencontre entre un chercheur et un individu. Dans nombre de manuels étudiés, tout se passe comme si le réel était traduisible et transmissible im-médiatement, les acteurs étant des objets ou des matériaux, l'observation et l'écriture étant dépourvues d'épaisseur propre. Ainsi, dans le Manuel de recherche en sciences sociales, les "hommes ordinaires" se voient attribuer plusieurs statuts différents, mais restent cantonnés dans un univers très lointain de celui du chercheur. Ils sont acteurs, témoins ou intermédiaires, mais en tous cas servent au chercheur de voie d'accès, passive, à l'information. La méthode consistant souvent à considérer les acteurs non comme des sujets mais comme porteurs des structures du social, en quelque sorte des réservoirs d'information. On voit que ce qui se joue derrière ce traitement du réel et des acteurs rencontrés dans la recherche est une conception des objets de la recherche comme "matériau ", c'est-à-dire une matière inerte à travailler, aux antipodes d'une élaboration de la co-construction entre chercheur et objet. Celle-ci s'exprime notamment par des séries de métaphores, par exemple policières dans L'entretien compréhensif, où on imagine des « mobiles » et où on recueille des « preuves ».

\section{La poursuite de la situation « pure »}

10 Cette conception des objets comme matériaux conduit aussi à minimiser ou ne pas aborder les modalités pragmatiques des échanges, tendance particulièrement visible dans le traitement des techniques d'entretien et d'observation. A cet égard, on constate dans notre corpus peu de différences entre les manuels qui se concentrent particulièrement sur les entretiens et les autres. Ils ont en commun d'opposer les biais occasionnés par le dispositif d'entretien et le caractère « pur » de la situation avant la mise en place d'un tel dispositif. C'est dire qu'aussi bien pour le Manuel de recherche en sciences sociales, que pour L'entretien, toute intervention du chercheur sur son terrain est nécessairement un biais et une distorsion du réel, la situation observée perdant de sa prétendue pureté. Cette poursuite de la «situation pure » pousse certains manuels, comme L'entretien compréhensif , ou le Guide de l'enquête de terrain, à une théorisation particulière de l'entretien, qui doit mimer la familiarité et le style oral quotidien pour être le plus proche possible des échanges ordinaires, du dialogue "vrai». Les "résistances» des interviewés seraient ainsi des obstacles qu'une bonne technique parviendrait à faire ployer. "L'observation directe doit vaincre la résistance ou l'inertie des individus », peut-on lire dans le Manuel de recherche en sciences sociales.

11 Dans cette quête d'un social présenté comme plus authentique quand il n'est pas observé, les échanges entre ce qu'il est convenu d'appeler l'enquêteur et ses « enquêtés » revêtent un caractère paradoxal. Le Guide de l'enquête de terrain, présentant la méthode de l'observation participante et de l'entretien de type ethnologique, alors même qu'il explique les enjeux liés à la construction du réel et à la rencontre avec les acteurs, décrit 
les entretiens comme des moments particuliers soumis à un protocole précis. Présentés en différentes étapes, les entretiens apparaissent d'abord comme des entretiens exploratoires pour devenir des entretiens informatifs et enfin des entretiens de vérification. Au sein même de la rencontre, et alors que le manuel définit l'entretien comme un moment d'institutionnalisation du moi dans lequel il serait possible de lire la manière dont la personne interviewée se construit une identité de circonstance, le chercheur doit "conduire l'échange", "ne pas induire de réponse ». L'art de l'enquête explique ainsi qu'un entretien (même par questionnaire) est un acte de communication et une "co-construction du monde", mais ne s'intéresse qu'aux signes qui vont de l'interviewé vers l'interviewer et non l'inverse, et maintient par ailleurs l'idée du recueil d'une information qui serait déjà là dans les phénomènes. Dans l'ensemble, les manuels les plus réflexifs mais aussi certains classiques comme le Manuel de recherche en sciences sociales présentent les situations d'échanges et d'interactions dans toute leur complexité communicationnelle mais maintiennent par ailleurs la fiction d'un social pur, acommunicationnel, et observable en dehors de la communication. D'où, chez ce dernier par exemple, la préférence affirmée pour les méthodes d'observation « plus directes » ou plus masquées, considérées comme restituant plus adéquatement l'authenticité du social.

Quand elle est perçue, la dimension communicationnelle l'est donc négativement, comme bruit à éradiquer ou contrôler, plutôt que positivement comme un outil qui non seulement permet d'approcher les objets, mais aussi de faire émerger des enjeux. Ce traitement de la communication, variable selon les manuels étudiés, engage bien sûr différentes dimensions constitutives de ceux-ci: notamment leur conception de la méthode, et leur positionnement par rapport à des traditions disciplinaires et épistémologiques, mais aussi les différentes postures et positionnements scientifiques des auteurs ainsi que les stratégies énonciatives et éditoriales qu'ils adoptent.

\section{Discursivité des manuels et performance de l'épistémologie}

13 Les manuels n'ont certes pas pour vocation l'élaboration épistémologique, ils n'en sont pas moins porteurs de modèles épistémologiques implicites, repérables par une analyse sémio-discursive précise. L'attention portée à leur composition, à leur mode d'énonciation, et leur régime polyphonique montre bien qu'ils instituent une conception de la communication. Qu'en est-il de la communication de la méthode ? Y a-t-il un lien entre le statut donné à la communication dans la présentation de la recherche et la manière de la communiquer, ou, en d'autres termes, entre le style de la méthode et celui du manuel ? Dans quelle mesure l'épistémologie sous-jacente à l'écriture de ces manuels coïncide-t-elle avec l'épistémologie à l'œuvre dans la présentation de la recherche?

\section{Communication de la méthode et scénarisation de la recherche}

Qu'il s'agisse de rendre compte de l'élaboration d'un questionnement intellectuel ou des modalités pratiques de mise en œuvre de la recherche, les manuels tendent à segmenter le travail en une succession de séquences, paradoxalement hermétiques les unes aux autres. Le Manuel de recherches en sciences sociales structure par exemple la recherche en sept " étapes " ${ }^{3}$ bien circonscrites. De manière comparable, le Guide du mémoire distingue des « phases essentielles dans le travail du mémoire $»^{4}$. Ce découpage est soutenu par une 
rhétorique programmatique allant de la numérotation à la mise en scène d'un calendrier de travail, en passant très souvent par des représentations schématiques. Certaines descriptions atteignent parfois une dimension véritablement dramaturgique comme dans L'entretien compréhensif qui file la métaphore théâtrale jusqu'à la présentation de la dernière étape qualifiée "d'acte final ». Des phases, non traitées spécifiquement dans le manuel, peuvent, par le biais de ce séquençage généralisé, être rejetées dans un ailleurs textuel. C'est entre autres le cas dans L'enquête et ses méthodes: l'entretien qui renvoie le lecteur soucieux d'élaborer sa problématique au Manuel de recherches en sciences sociales, un classique qu'on appelle généralement, du nom de ses auteurs, "le Quivy et Campenhoudt ». Le processus de cloisonnement est d'ailleurs à son comble dans ce manuel : « une fois les hypothèses formulées, le choix de l'enquête arrêté, son objectif et sa fonction dans le dispositif de recherche définis, se pose la question de savoir qui interroger et dans quelle population $»^{5}$.

Cette forme de scénarisation, commune à presque tous les manuels de notre corpus, tend à lisser le processus de recherche et à réduire toutes perturbations, hésitations ou mouvements de va et vient d'une "étape " à l'autre. Cette logique ne relève-t-elle pas d'abord des contraintes de linéarité textuelle à laquelle sont évidemment soumis les manuels? Le lissage accompli dans L'enquête et ses méthodes: l'entretien correspond à la présentation académique et rassurante du manuel lui-même. La mise en intrigue de la recherche, séquence par séquence, rappelle toutefois la manière dont certains chercheurs décrivent leur propre travail, ce qui permet de s'interroger sur la dimension instituante des manuels de méthode. Cela tend à produire non pas un mais des processus de recherche cloisonnés qui, de ce fait, ne communiquent pas entre eux. Il y a là sans doute un moyen de lever partiellement le paradoxe de la prise en compte de la communication. Une attention très poussée à la situation de rencontre entre le chercheur et les acteurs au moment de la présentation de la méthode de l'entretien n'entraîne pas l'application de la même précaution lors de «l'étape de l'exploitation », ces moments pouvant êtres conçus et abordés de manière autonome.

La logique du scénario semble finalement masquer la réalité du passage du terrain au texte. Elle garantit surtout une forme de reproductibilité du protocole de recherche, implicitement considérée comme une condition de sa scientificité.

\section{Postures auctoriales et postures scientifiques}

Les critères de scientificité de cette épistémologie latente sont à lire à d'autres niveaux dans les manuels. Une analyse précise des différents contrats énonciatifs portés par les ouvrages choisis permet de décrire plusieurs relations instaurées entre l'auteur, en tant qu'il est construit par le texte, et le lecteur visé : relation pédagogique qui associe un professeur à des étudiants, relation initiatique dans laquelle se rencontrent plutôt un maître et un disciple, relation autoritaire... Il nous semble plus intéressant d'indiquer dans quelle mesure se dessinent dans ces configurations textuelles de véritables postures scientifiques. Derrière l'énonciateur du discours de la méthode se profile en effet très souvent le chercheur ou du moins le spécialiste qui dispense ses conseils ou donne ses "ficelles". Les modes d'énonciations renforcent généralement le statut accordé au chercheur dans la description du processus de recherche. Howard Becker dans Les ficelles du métier ou J. C. Kaufman dans L'entretien compréhensif assument, par exemple, une énonciation à la première personne et réalisent dans leurs manuels une sorte 
d'autoportrait de l'auteur en chercheur, plaçant au cœur de la recherche l'individu qui la conduit. Le modèle de chercheur qui se dégage à la fois de l'écriture du manuel et de la méthode qu'il propose est celui de l'aventurier passionné, inventif, créatif dont la rigueur scientifique relève surtout de la mise en œuvre d'une réflexivitét. Le « manuel d'auteur " célèbre ici la construction d'une véritable posture auctoriale dans la recherche. A l'inverse, dans d'autres manuels comme L'enquête et ses méthodes: l'entretien, les auteurs ont choisi un mode d'énonciation beaucoup plus distancié pour présenter la «technique de l'entretien » de façon panoramique et transdisciplinaire. La méthode semble garante en elle-même de la scientificité du travail. L'impersonnalité du manuel conforte une conception de la recherche dans laquelle l'interchangeabilité des chercheurs renvoie à celle de leurs objets, considérés comme des reflets de leurs groupes sociaux. Dans Le sociologue et son terrain. Trente ans de recherches exemplaires, les auteurs se citent euxmêmes sur un mode impersonnel. Cette distance sert la mise en scène d'une véritable isotopie du bricolage, aussi bien dans la conception même de la recherche que dans le manuel lui-même, ici construit comme un recueil de textes d'auteurs. Le lecteur y est invité à « ouvrir une boîte noire » et à « observer le sociologue dans son atelier [...] choisir ses outils en lien avec les objectifs de la recherche ». Le manuel s'institue de fait comme une boîte à outils dans lequel le chercheur inexpérimenté sera sûr de trouver son instrument d'observation et d'analyse adéquat, par l'intermédiaire du florilège de grands textes de sociologie.

Les manuels de méthode s'inscrivent par leur énonciation même dans des configurations qui tracent les contours d'une épistémologie des sciences humaines et sociales. Les postures auctoriales sont de plus à mettre en lien avec des positionnements éditoriaux - il existe par exemple des collections spécialisées dans les manuels - mais surtout avec des positionnements disciplinaires qui renvoient à des univers épistémologiques distincts.

\section{Espace polyphonique et enjeux disciplinaires}

19 Si l'un des éléments définitoires de tout manuel est qu'il se désigne explicitement comme manuel, cette explicitation est bien souvent l'occasion de se présenter comme « différent des autres ». On peut voir dans cette volonté démarcative une invitation à prendre en compte dans notre analyse les liens tissés non seulement à l'intérieur de chaque discours de la méthode avec d'autres discours du même genre, mais aussi l'ensemble des renvois, citations et références qui délimitent un véritable espace communicationnel.

20 Cet espace est tout à fait comparable à celui délimité par les textes du monde de la recherche ${ }^{7}$. La manière dont des conventions polyphoniques spécifiques (notes de bas de pages, citations, bibliographies...) sont présentes dans les manuels parfait leur inscription dans le champ scientifique. Nous retrouvons dans ces deux réseaux textuels des modes de légitimation et de positionnement similaires.

21 Aussi l'étude des pratiques citationnelles révèle-t-elle que des enjeux disciplinaires, souvent non revendiqués en tant que tels, travaillent la belle objectivité des manuels de méthode. Au détour des allégations, c'est toute une hiérarchie des auteurs et des disciplines qui s'y trouve organisée. Certains auteurs, ailleurs incontournables, disparaissent de certaines bibliographies, tandis qu'on assiste à de véritables hagiographies de chercheurs dont la place est problématique pour qui veut voir dans le manuel un outil méthodologique et non pas une machine à légitimer. Ces mouvements polyphoniques jouent certainement un rôle, en ce qu'ils contribuent à ordonner les 
disciplines entre elles, voire à nourrir les enjeux d'institutionnalisation de certaines. Alors que les manuels généralistes prennent comme référence principale la sociologie dans les méthodes explicitées comme dans les ouvrages cités, les manuels spécialisés, eux, s'inscrivent dans des disciplines spécifiques qui revendiquent chacune la paternité ou l'utilisation de certaines méthodologies.

À cet égard, les rapports qu'entretiennent la sociologie et l'ethnologie à travers leurs manuels respectifs sont symptomatiques de cet agencement agonistique. Ainsi, dans Le sociologue et son terrain, si le dialogue entre les deux disciplines est le plus souvent traité de manière neutre, quelques raccourcis peu flatteurs subsistent: "l'observation participante est la version de la démarche ethnographique dans les sociétés urbaines. L'ethnologue s'installe pour de longs mois dans son village ou sa tribu pour participer à la vie sociale commune et l'observer à travers cette participation : on ne peut observer la vie d'un petit groupe humain sans en faire partie. Dans les sociétés modernes, la distance entre l'observateur et l'observé est moins grande: le sociologue participe à la même société que les sujets observés qu'en s'y faisant admettre ». C'est toute la thématique du grand partage qui est ici convoquée: aux ethnologues les tribus lointaines, aux sociologues les sociétés proches. Cette délimitation se retrouve au niveau des références convoquées et de la pratique de la citation ou de la bibliographie: aucun ouvrage d'anthropologie dite du contemporain ne sera présenté dans ce manuel.

Ce niveau interprétatif permet de relire différemment les modalités de prise en charge de la communication par les manuels et de comprendre comment des enjeux disciplinaires s'inscrivent dans leur espace. La discipline de référence dirige fortement vers un type de traitement : les manuels se réclamant de la psychosociologie évacueront radicalement les aspects communicationnels ; ceux issus de l'anthropologie lui feront la plus grande place. Si la sociologie occupe une position médiane dans ces configurations, c'est aussi la discipline qui rayonne le plus : la manière dont elle traite les dimensions interactionnelles de la recherche est largement reprise et diffusée par les manuels généralistes. Il n'en va pas de même pour l'hyperconscience communicationnelle d'un ethnologue comme Jean Copans, qui trouve un écho beaucoup moins important dans le petit monde des manuels de recherche.

Lieux de passage obligés dans la formation à la recherche en sciences humaines et sociales, les manuels de méthode sont un espace privilégié d'institution d'un certain statut de la communication: jamais élevée au rang de problème épistémologique, elle n'en reçoit pas moins un traitement spécifique, qu'une prise au sérieux de l'objet manuel dans toute son épaisseur sémio-discursive a permis de caractériser. L'analyse de la communication dans la méthode, mettant en évidence les logiques de convocation et de conjuration de l'épaisseur interactionnelle de tout processus de recherche ne peut donc se penser sans une analyse de la communication de la méthode qui, elle, a repéré différentes configurations possibles de la prise en compte de cette incontournable absente.

Reste que, si les manuels de recherche et autres ouvrages de méthode peuplent les rayons des librairies et des bibliothèques, c'est à présent à une analyse des usages que la démarche engagée doit conduire. Car la prise en compte des usages des manuels permettra sans doute de déterminer la portée effective des mécanismes instituants ici décrits. 


\section{BIBLIOGRAPHIE}

Althabe, G., (1990), « Ethnologie du contemporain et enquête de terrain », Terrain, nº14.

Berthelot, J.-M., (dir.), (2001), Epistémologie des sciences sociales, Paris, P.U.F.

Bourdieu, P., (1993), « Comprendre », postface, dans La Misère du monde, Paris, Seuil.

Compagnon, A., (1979), La seconde main ou le travail de citation, Paris, Seuil.

Devereux, G., (1980), De l'angoisse à la méthode dans les sciences du comportement, Paris, Aubier.

Kilani, M., (1994), « Du terrain au texte », Communication, $\mathrm{n}^{\circ} 58$.

Le Marec, J., (dir.), (2002), « Questions de terrain », Études de communication, n²5.

Latour, B., (1989), La science en action, Paris, La Découverte.

Olivesi, S., (1997), « De l'anthropologie critique à l'épistémologie de la communication. Variations critiques autour de Palo Alto », Réseaux, n²5.

Revel, J., (dir.), (1996), Jeux d'échelles. La micro-analyse à l'expérience, Paris, Gallimard-Seuil.

Schutz, A., (1987), Le chercheur et le quotidien, Paris, Méridiens-Klincksieck.

\section{NOTES}

1. Ce questionnement sur les manuels est né au sein d'un groupe interdisciplinaire de chercheurs tous chargés, notamment, d'enseignement de méthodologie de la recherche en SIC. Le groupe de recherche " épistémologie des SIC et des SHS » du GRIPIC, Université Paris IV - Sorbonne - CELSA est composé de Cécile Deparis, Lucile Desmoulins, Anne Jarrigeon, Yves Jeanneret, Sarah Labelle, Emmanuelle Lallement, Florent Loiseau, Joëlle Menrath, Jean Baptiste Perret et Julien Tassel.

2. Ce texte s'appuie sur une analyse de manuels (récents ou classiques réédités) réalisée en 2001-2002 par les membres du groupe "épistémologie des SIC et SHS ». Le corpus est composé de: L'art de l'enquête, guide pratique, Yannis Harvatopoulos, Yves-Frédéric Livian et Philippe Sarnin, Paris: Eyrolles, 1989, Exercices corrigés: méthodes d'enquête en psychosociologie, Hélène Chauchat, Dunod, 1996, Paris, L'enquête et ses méthodes : l'observation directe, Anne-Marie Arborio et Pierre Fournier, Nathan, 1999, Paris, L'entretien compréhensif, Jean-Claude Kaufman, Nathan, 1996, Paris, Le sociologue et son terrain: trente recherches exemplaires, Henri Mendras, Marco oberti et Armand Colin, 2000, Paris, Guide de l'enquête de terrain, Stéphane Beaud et Florence Weber, La Découverte, 2003, Paris, L'enquête ethnologique de terrain, Jean Copans, Nathan, 1999, Les ficelles du métier, Howard Becker, La découverte, 2002, Paris, Manuel de recherches en sciences sociales, Raymond Quivy et Luc Van Campenhoudt, Dunod, 1988, Paris, Le mémoire de recherche en information-communication, Jean-Luc Michel, Ellipses, 1999, Paris, Guide du mémoire, Anne-Sophie Andreu et Aldo Lévy, Gualino, 1998, Paris, Méthodologie des pratiques de terrain en SHS, Joël Guibert, Guy Jumel et Armand Colin, 1997, Paris.

3. Les ouvrages retenus pour ce travail correspondent tous, malgré une forte disparité apparente, à quelques critères minimaux de définition : ils se désignent explicitement, dans leur discours et dans leur forme, comme des manuels, ils sont publiés dans une collection de manuels, ils s'adressent à des étudiants avec une visée d'apprentissage. Cette définition du manuel permet de 
prendre en compte des textes très différents : des ouvrages regroupant des textes canoniques faisant office de manuel, jusqu'aux manuels classiques comme le Manuel de recherche en sciences sociales, dit aussi le "Quivy et Campenoud», en passant par des manuels plus ciblés, présentant une technique particulière (l'entretien, l'observation...) et des manuels à visée plus pragmatique, se fondant sur la production d'un document écrit de type mémoire, voire thèse.

4. Les étapes, reprises à plusieurs endroits du manuel, de la table des matières à l'annonce du plan, sont : « la question de départ », « l'exploration », « la problématique », « la construction du modèle d'analyse ", « l'observation ", « l'analyse des informations », « les conclusions ».

5. Ce manuel accorde une place plus importante à la dimension pratique de la réalisation d'un mémoire. Les phases distinguées sont liées à cette visée plus opérationnelle: "la phase d'exploration, la phase de recherche, la phase de composition, la phase de rédaction, la soutenance "

6. L'enquête et ses méthodes : l'entretien, Gotman et Blanchet, op. cit., p. 23.

7. J. C. Kaufman revendique clairement la figure « de l'artisan intellectuel ».

\section{RÉSUMÉS}

L'enseignement des méthodes en sciences humaines se traduit éditorialement par un nombre croissant de publications de type «manuels ». Pour qui s'intéresse à l'épistémologie des sciences humaines, ces manuels représentent une véritable scène d'exposition, de cristallisation, et de modélisation des pratiques de recherche. Il s'agit ici d'analyser comment les «discours de la méthode » intègrent et manipulent, à des degrés et selon des modalités diverses, les dimensions communicationnelles inhérentes à toute recherche. Alors qu'on assiste à un mouvement de prise en compte croissant de l'épaisseur de l'interaction communicationnelle entre le chercheur et les autres acteurs sociaux, le discours méthodologique, de par sa vocation, se trouve pris dans une position contradictoire : s'ouvrir à la complexité des enjeux communicationnels de la recherche (caractère ouvert des situations d'interaction, attitudes de relation aux objets, formes matérielles des documents, rhétorique d'exposition, etc.) tout en réaffirmant les principes d'une épistémologie classique qui assimile la scientificité des procédures à l'extériorité du chercheur vis-à-vis de ce qu'il étudie. Ainsi, de moins en moins «incontournable », la communication demeure-t-elle un objet à conjurer plus qu'à intégrer.

Teaching the methods used in the social sciences tends to generate an increasing number of publications of the textbook type. For those who are interested in the epistemology of the social sciences, these books offer an arena in which research practices are exhibited, crystallized, and modeled. The aim of this paper is to analyze how discourses on methodology assimilate the communication aspects inherent in any research, at many levels, and according to various modalities. While there is a trend toward taking increasing account of the depth of communicative interactions between researchers and other social players, by its very nature, methodology finds itself in a contradictory position. That is, it must become aware of the communication challenges presented by research (including the open nature of interactive situations, relationships with objects, the material forms of documents, and expository rhetoric) and at the same time reassert the principles of a classical epistemology that compares the scientific character of practices to the exteriority of researchers toward what they study. Thus, 
although communication is becoming less and less inescapable, it remains an object that must be averted rather than assimilated.

INDEX

Mots-clés : communication, épistémologie, manuels de méthode, méthodologie

Keywords : communication, epistemology, hand books, methodology

\section{AUTEURS}

ANNE JARRIGEON

GRIPPIC - CELSA - Université de Paris IV - Sorbonne

\section{EMMANUELLE LALLEMENT}

GRIPPIC - CELSA - Université de Paris IV - Sorbonne

JEAN-BAPTISTE PERRET

GRIPPIC - CELSA - Université de Paris IV - Sorbonne

JULIEN TASSEL

GRIPPIC - CELSA - Université de Paris IV - Sorbonne 